\title{
Contemporary Classification of Glucagon-Like Peptide 1 Receptor Agonists (GLP1RAs)
}

\author{
Sanjay Kalra $\cdot$ Saptarshi Bhattacharya $\cdot$ Nitin Kapoor
}

Received: May 24, 2021 / Accepted: July 1, 2021 / Published online: July 15, 2021

(C) The Author(s) 2021

\section{ABSTRACT}

This communication provides a contemporary classification of glucagon-like peptide 1 receptor agonists (GLP1RAs) based on indication, route, and frequency of administration, which could support a person-centric approach to treatment choice. It includes all recently developed GLP1RAs as well as those in advanced stages of clinical study. Keeping pace with current trends in pharmacology and metabolic medicine, it attempts to bring clarity and simplicity to a complex spread of information.

S. Kalra

Department of Endocrinology, Bharti Hospital,

Karnal, India

S. Bhattacharya

Department of Endocrinology, Max Superspeciality

Hospital, Patparganj, New Delhi, India

N. Kapoor $(\square)$

Department of Endocrinology, Diabetes and Metabolism, Christian Medical College, Vellore, India

e-mail: nitin.kapoor@cmcvellore.ac.in

\section{N. Kapoor}

Non Communicable Disease Unit, Melbourne School of Population and Global Health, University of Melbourne, Victoria, Australia
Keywords: Dulaglutide; Danuglipron; Exenatide; Exenatide LAR; GLP1RA; IDegLira; IcoSema; Liraglutide; LixiLan; Person-centric care; Semaglutide; Tirzepatide

\section{Key Summary Points}

This review summarizes the latest developments in GLP1RA therapy for the management of diabetes and obesity.

All recently developed GLP1RAs, as well as those in advanced stages of clinical study, are described.

In addition to GLP1RA monotherapies, various additional GLP-1RA coadministration strategies are discussed, citing the most recent evidence available for them.

A contemporary classification of GLP1RAs based on indication, route, and frequency of administration is also provided.

\section{INTRODUCTION}

The glucagon-like peptide 1 receptor agonists (GLP1RAs) are a modern, rapidly evolving class of drugs for which there are an increasing 
number of indications. GLP1RAs were originally only available as injectable therapies for the glycaemic management of type 2 diabetes mellitus (T2DM), but they are now also available in oral formulations, such as oral semaglutide, in several countries. These molecules are currently being studied in relation to their possible use for various other indications, such as for cardiovascular risk reduction in T2DM, for weight reduction in obesity, for nonalcoholic steatohepatitis (NASH), and for Alzheimer's. Recent advances in GLP1RA pharmacology have created a need to revisit the classification of this class of drugs. This article is based on previously conducted studies and does not contain any new studies with human participants or animals performed by any of the authors.

\section{EARLIER CLASSIFICATIONS}

Earlier taxonomic frameworks were based on the structure of the GLP1RA (exendin-based and human glucagon-like peptide-1 (GLP-1)). However, this taxonomic basis does not aid clinical decision making. The recent expansion in indications for the administration of GLP1RAs is not reflected in earlier classification schema. The South Asian Task Force provides consensus recommendations on the use of GLP1RAs; it classifies GLP1RAs according to their duration of action into short, intermediate, long, and continuous acting.

\section{IDEAL CLASSIFICATIONS}

An ideal taxonomic structure should help the user in his or her daily work. For a student of pharmacology, for example, an ideal classification should describe drug pharmacokinetics and dynamics. For the clinician, a rubric should assist with the appropriate use of the concerned drugs. A person-centric approach, crafted with the person seeking medical care in mind, is perhaps the best way to craft and organize a drug classification system.

GLP1RAs have been listed as acting on fasting, postprandial, or both forms of hyperglycaemia. This approach has, however, been criticized for being myopic and oversimplistic. While duration of action is a pharmacological contract, frequency of administration is personoriented, and has been used to create a personcentred taxonomy for GLP1RAs [1].

\section{CONTEMPORARY CLASSIFICATION}

We suggest a contemporary classification scheme for GLP1RAs that incorporates drug indication, route of administration, and frequency of administration. This GLP1RA classification is concordant with the thought process and conversational flow of a typical patient-provider interaction: determine the indication, achieve agreement on the preferred route of administration, and then choose from various options.

Some GLP1RA preparations have been withdrawn from the market while others are in advanced stages of development. We have chosen to include these for the sake of completeness. GLP1RAs are also available as co-formulations with insulins, and are being studied as co-formulations with other peptide agonists. These, too, are included in this review to ensure comprehensive coverage.

Table 1 summarizes the proposed classification based on route and indication. We hope that this person-centric, reader-friendly classification of GLP1RA will benefit students, clinicians, and researchers alike.

\section{CLASSIFICATION OF GLP1RAS BASED ON INDICATION}

Broadly speaking, we can classify GLP1RAs based on whether they are indicated for (or, if the GLP1RA is in the development phase, intended for) T2DM or for obesity.

\section{GLP1RAs for the Treatment of T2DM}

GLP1RAs are becoming a popular treatment option for people with T2DM due to their multiple benefits, such as their ability to lower glycated haemoglobin levels and reduce weight and their low risk of causing hypoglycaemia 
Table 1 Contemporary classification of glucagon-like peptide 1 receptor agonists (GLP1RAs)

\begin{tabular}{|c|c|c|}
\hline Route/indication & Type 2 diabetes mellitus & Obesity \\
\hline \multicolumn{3}{|l|}{ Monoformulations } \\
\hline \multirow[t]{3}{*}{ Oral } & Semaglutide $3,7,14 \mathrm{mg}$ & Semaglutide $50 \mathrm{mg;}{ }^{a}$ \\
\hline & Semaglutide $25,50 \mathrm{mg}^{\mathrm{a}}$ & Danuglipron $^{\mathrm{a}}$ \\
\hline & Danuglipron $^{a}$ & \\
\hline Subcutaneous daily & Lixisenatide; liraglutide $0.6-1.8 \mathrm{mg}$; exenatide $\mathrm{BD}$ & Liraglutide $3.0 \mathrm{mg}$ \\
\hline Subcutaneous weekly & Exenatide LAR; dulaglutide; semaglutide $0.25-1.0 \mathrm{mg}$; albiglutide ${ }^{\mathrm{b}}$ & Semaglutide $2.4 \mathrm{mg}$ \\
\hline Implantable & ITCA $650^{\mathrm{b}}$ & \\
\hline \multicolumn{3}{|l|}{ Co-formulations } \\
\hline Route/content & GLP1RA + insulin & GLP1RA + other agonist \\
\hline Daily subcutaneous & IDegLira; LixiLan & \\
\hline \multirow[t]{3}{*}{ Weekly subcutaneous } & IcoSema $^{a}$ & Sema-Amylin ${ }^{a}$ \\
\hline & & $\mathrm{HM} 15211^{\mathrm{a}}$ \\
\hline & & Tirzepatide $5,10,15 \mathrm{mg}^{\mathrm{c}}$ \\
\hline
\end{tabular}

(Table 2). Apart from these advantages, cardiovascular outcome trials (CVOTs) of GLP-1RA conducted over the years have established the cardiovascular $(\mathrm{CV})$ safety of GLP1RAs, as they are noninferior to placebo in cardiovascular outcomes, whereas some GLP1RAs such as liraglutide have shown CV superiority over placebo in reducing three-point major adverse cardiovascular events (MACEs) [2]. Despite their shared overall mechanism, a wide range of clinical results and pharmacodynamics are shown by GLP1RAs because they vary significantly in structure and pharmacokinetics [3]. In this review, we attempt to describe each briefly from the clinician's point of view.

\section{Exenatide Injection BID}

Exendin-4 is a peptide present in the saliva of the Gila monster [4]. Exenatide is a synthetic form of exendin-4 that has $50 \%$ sequence homology with the native GLP-1 hormone. It was first approved in 2005 for the treatment of T2DM. Substitution of the amino acid arginine with glycine at position 2 makes exenatide resistant to the enzyme dipeptidyl peptidase- 4 (DPP-4) and provides a half-life of around $2-4 \mathrm{~h}$ [5]. A twice-daily dose regime administered 60 min before breakfast and dinner caused glycated haemoglobin $\left(\mathrm{HbA}_{1 \mathrm{c}}\right)$ levels to drop by approximately $1.0 \%$ over 30 weeks and a weight loss of $1.0-2.5 \mathrm{~kg}$ over 16-30 weeks [6]. Exenatide once-weekly also led to a $12 \%$ reduction in triglyceride, a $6 \%$ reduction in low-density lipoprotein C (LDL-C), and a $24 \%$ increase in high-density lipoprotein C (HDL-C), resulting in an overall improvement in the lipid profile [7]. A systemic review found that exenatide and insulin produced similar reductions in $\mathrm{HbA}_{1 \mathrm{c}}$, but in terms of weight reduction, exenatide excelled over insulin by inducing a loss of $3-6 \mathrm{~kg}$ at 52 weeks of follow up. Although hypoglycaemia rates were similar with exenatide and insulin, placebo-controlled trials have shown that nausea is a major adverse event in patients treated with exenatide [6]. Increasing the dose of exenatide in patients with an estimated glomerular filtration rate 
(eGFR) of $30-60 \mathrm{~mL} / \mathrm{min} / 1.73 \mathrm{~m}^{2}$ is not recommended, and it is contraindicated in patients with an eGFR of less than $30 \mathrm{~mL} / \mathrm{min} /$ $1.73 \mathrm{~m}^{2}$.

\section{Exenatide Extended Release (ER) QW}

A sustained-release formulation of exenatide consisting of microspheres of exenatide and a biodegradable medical polymer was developed as an exenatide once-weekly injection. Exenatide QW has been shown to produce a significantly greater reduction in $\mathrm{HbA}_{1 \mathrm{c}}$ than exenatide BID, whereas weight reduction benefits and hypoglycaemic event rates were comparable [4]. Since exendin-4 is a foreign element used in the development of exenatide, it is expected to cause antibody formation in humans. A post-hoc analysis confirmed that there was low-titre antiexenatide antibody formation in humans with both exenatide BID and exenatide QW, but this had no effect on the efficacy of exenatide. However, higher-titre antibody formation-a less common phenomenon-leads to a significant reduction in the average efficacy of exenatide QW [8].

\section{Lixisenatide}

The exendin-4-based GLP1RA lixisenatide has a half-life of $2-3 \mathrm{~h}$ with a once-daily dose injection regimen [9]. The GetGoal clinical trial programme for lixisenatide showed that $\mathrm{HbA}_{1 \mathrm{c}}$ was lowered by up to $0.9 \%$, and there was a marked reduction in postprandial glucose (PPG), probably due to the delaying effect of lixisenatide on gastric emptying [10]. In terms of weight loss, lixisenatide induced a reduction of $1-3 \mathrm{~kg}$ [11]. Since it is derived from a foreign peptide, antibody production after the administration of lixisenatide to patients is frequently seen [9].

\section{Liraglutide}

Liraglutide-administered as a once-daily injection-is a GLP1RA with two minor structural differences from endogenous glucagonlike peptide-1 (GLP-1) that lead to 97\% homology with the GLP-1 sequence. A C-16 fatty acid is attached with the help of a glutamine spacer to the lysine at position 26 in the amino acid chain, and the lysine at position 34 is replaced with arginine. These minor structural changes lead to a half-life of $13 \mathrm{~h}$, slow dissolution of liraglutide from the injection site, increased binding to albumin, and reduced inhibition by DPP-4 [12]. The immunogenic reaction (the development of antiliraglutide antibodies) induced by liraglutide is less than that induced by exenatide and has no impact on its efficacy and safety [13]. The Liraglutide Effect and Action in Diabetes (LEAD) programme, which focused on the effects of liraglutide at doses of 1.2 and $1.8 \mathrm{mg}$, demonstrated the superiority of this GLP1RA for glycaemic control and weight loss compared with active comparators or placebo. Liraglutide also reduces the systolic blood pressure (SBP) and improves beta-cell function and the lipid profile [14]. The Liraglutide Effect and Action in Diabetes: Evaluation of Cardiovascular Outcome Results (LEADER) CVOT trial showed the superiority of liraglutide by noting that its use yielded a 13\% MACE reduction [15].

\section{Once-Weekly Semaglutide (Semaglutide QW)}

Semaglutide is an acylated GLP1RA, like liraglutide. It has 94\% homology with GLP-1. It differs from GLP-1 in two amino acids $(\alpha$ aminoisobutyric acid (Aib) at position 8 and arginine at position 34) and the addition of a C-18 diacid to the lysine at position 26 , which allow the utilization of semaglutide as a onceweekly injectable therapy [16]. The phase 3a clinical development programme SUSTAIN established the superiority of semaglutide for $\mathrm{HbA}_{1 \mathrm{c}}$ reduction and weight reduction in comparison to placebo as well as active comparators [17]. SUSTAIN 6, a CVOT of semaglutide QW, confirmed its cardiovascular safety as well as noting a significant $(26 \%)$ reduction in MACEs [18]. SUSTAIN 1-5 did not find an increase in diabetic retinopathy (DR) events with semaglutide QW use, but a significantly increased (by $76 \%$ ) risk of DR was seen in the SUSTAIN 6 trial [19].

\section{Dulaglutide}

Dulaglutide, a GLP1RA with a half-life of 4 days, comes as a once-weekly dosage injection. Its ability to bind with human IgG4 keeps it 
protected from proteolytic degradation by DPP4. Moreover, its high molecular weight (57 kDA) prevents its renal clearance and extends its halflife [20]. Compared with controls such as placebo, metformin, and liraglutide, dulaglutide results in significant reductions in $\mathrm{HbA}_{1 \mathrm{c}}$ and fasting plasma glucose (FPG), a similar risk of hypoglycaemia, and less body weight loss when used as a monotherapy [21]. In all phase 3 studies of dulaglutide (the AWARD studies), dulaglutide enabled significantly more patients to achieve their $\mathrm{HbA}_{1 \mathrm{c}}$ targets than when other comparators (except liraglutide) were used [22]. A MACE reduction of $12 \%$ was reported from a CVOT of dulaglutide (REWIND) [23].

\begin{abstract}
Albiglutide
Another long-acting GLP1RA with once-a-week dosing, albiglutide is a GLP-1 dimer bound to albumin and has notable DPP-2 resistance. This agent produces fewer gastrointestinal tract (GIT) side effects than other comparable GLP1RAs and is safe in patients with renal failure. In comparison to several other GLP1RAs, it produces a smaller improvement in $\mathrm{HbA}_{1 \mathrm{c}}$ : up to $1 \%$. Its weight reduction benefits are also less profound compared to other GLP1RAs [24]. This could be due to its relatively large molecular weight and reduced central nervous system (CNS) penetration [25].
\end{abstract}

\section{Oral Semaglutide}

Semaglutide, a novel once-daily GLP-1 receptor agonist given orally as a tablet. The tablet formulation includes an absorption enhancer, sodium $\mathrm{N}$-(8-[2-hydroxybenzoyl]amino)caprylate (SNAC). This formulation was recently approved for the treatment of patients with T2DM [16]. The Peptide Innovation for Early Diabetes Treatment (PIONEER) clinical trial program investigated 3,7 , and $14 \mathrm{mg}$ doses of oral semaglutide, with $3 \mathrm{mg}$ used as the initial dose and 7/14 $\mathrm{mg}$ as the maintenance dose. In those studies, oral semaglutide provided significantly better efficacy than placebo and commonly used glucose-lowering medications, including DPP-4 inhibitors such as sitagliptin, sodium-glucose co-transporter-2 inhibitors (SGLT2is) such as empagliflozin, and the subcutaneous GLP1RAs liraglutide and dulaglutide. An event-driven pre-approval CVOT, PIONEER-6, showed that there was a $21 \%$ reduction in MACEs (noninferior) with oral semaglutide [26]. The efficacy and safety of high-dose (25 and $50 \mathrm{mg}$ ) oral semaglutide are currently being compared with those of $14 \mathrm{mg}$ oral semaglutide in an ongoing phase $3 \mathrm{~b}$ trial. This trial proposed to enrol 1224 people with T2DM who were previously treated with other oral antidiabetic medicines; it has a duration of 68 weeks and is expected to end by March 2023 [27].

\section{Danuglipron}

Danuglipron was developed in an attempt to obtain a small-molecule oral GLP1RA that could be used in standard formulations and could therefore be combined with other oral therapies, unlike oral semaglutide [28]. A phase I study was conducted to assess the safety profile, pharmacokinetics, and pharmacodynamics of the drug. Results from the study showed that $\mathrm{HbA}_{1 \mathrm{c}}$ was reduced by $0.9 \%, 1.2 \%$, and $1.2 \%$ at doses of 15,70 , and $120 \mathrm{mg}$, respectively, and that body weight was decreased by $4.0 \mathrm{~kg}$ (70 $\mathrm{mg}$ dose) and $7.9 \mathrm{~kg}$ (120 mg dose) after 4 weeks of treatment. The safety profile and tolerability of danuglipron were found to be similar to other GLP1RAs. Phase 2 clinical trials of this drug are ongoing [29].

\section{ITCA 650}

This is a miniature implantable device consisting of an osmotic pump system that maintains a continuous supply of GLP1RA by delivering subcutaneous exenatide at a constant preset rate for up to 12 months. Though invasive in nature, it provides freedom from repeated injection. However, its utility during illness, fasting, or unexpected changes in renal/hepatic parameters is thought to be one of its limitations [30].

\section{Co-formulations}

As T2DM is a chronic progressive disease leading to a gradual decline in $\beta$-cell function, treatment intensification through the use of a combination of drugs becomes necessary to 
address multiple pathophysiological defects $[31,32]$. Several clinical studies have shown that glycaemic control and weight gain can be improved without incurring a risk of hypoglycaemia by using basal insulin plus a GLP1RA; many such combinations are available and used [33], as described below.

GLP1RA and Insulin Combination Insulin is a logical partner for GLP1RAs, with an entirely complementary mode of action that leads to additive glucose reduction in subjects with T2DM. Therapies based on fixed-ratio combinations (FRCs) of a GLP1RA with basal insulin have been developed. Two insulin and GLP1RA co-formulated combinations have been approved to treat T2DM: lixisenatide-insulin glargine and liraglutide-insulin degludec. Both formulations produce robust glucose control, often without weight gain and with less hypoglycaemia than observed with insulin alone [34].

iGlar U-100/Lixisenatide 33 or $50 \mu \mathrm{g} / \mathrm{ml}$ (iGlarLixi) iGlar U-100/lixisenatide 33 or $50 \mu \mathrm{g} / \mathrm{ml}$ combinations used as a once-daily dose have been tested in LixiLan phase 2 and 3 trials. The studies demonstrated that iGlarLixi has superior efficacy for glycemic control and weight loss as compared to iGlar alone. Also, the risk of hypoglycaemia was not increased and the rates of nausea and vomiting were low in the iGlarLixi group [35].

Insulin Degludec $100 \mathrm{U} / \mathrm{ml}$ and Liraglutide $3.6 \mathrm{mg} / \mathrm{ml}$ (IDegLira) IDegLira is a fixed-ratio combination of basal insulin degludec $100 \mathrm{U} / \mathrm{ml}$ and liraglutide $3.6 \mathrm{mg} / \mathrm{ml}$ [36] that is administered as a once-daily injection at any time (albeit preferably at the same time) of the day. Improvements in both FBG and PPG along with reductions in hypoglycaemic events, weight gain, and nausea have been demonstrated in the DUAL I-VII trials. Therefore, this combination of ultralong-acting basal insulin and a GLP1RA utilizes the clinical benefits of both drugs [37].

\section{IcoSema (Insulin Icodec350U/Semaglutide $1 \mathrm{mg}$ ) IcoSema (insulin icodec350U/}

semaglutide $1 \mathrm{mg}$ ) is a novel FRC of onceweekly (OW) basal insulin icodec and semaglutide. It is recommended for onceweekly administration, irrespective of meals, in order to improve patient adherence and outcomes. It is expected to have superior $\mathrm{HbA}_{1 \mathrm{c}}$ reduction compared to monocomponents, weight benefits compared to basal insulin, and a reduced risk of hypoglycaemia. The phase 3 development program COMBINE $1-3$ is currently being conducted on this FRC [38].

GLP-1/GIP Receptor Dual Agonists Tirzepatide (formerly known as LY3298176) is a novel glucose-lowering medication that stimulates the receptors for both glucose-dependent insulinotropic polypeptide (GIP) and GLP-1. This 39-amino-acid peptide is based on the biologically active N-terminal GIP(1-14) sequence with an N2 aminoisobutyric acid substitution. The mid-sequence contains substitutions compatible with GLP-1 receptor agonism, and there is a C20 fatty diacid chain linked via glutamic acid to the Lys20 residue to facilitate attachment to albumin, prolonging viability in the circulation. There is also an amidated exenatide-like C-terminal sequence. The structure confers stronger agonism for the GIP receptor than the GLP-1 receptor: the binding affinity for the GIP receptor is similar to that of native GIP(1-42), but it is about fivefold weaker for the GLP-1 receptor than native GLP-1. This provides a degree of balance that takes account of the physiologically higher circulating concentrations of GIP than GLP-1. The modifications reduce enzymatic degradation and provide sufficient durability for once-weekly subcutaneous administration. Tirzepatide has been compared with placebo and other glucose-lowering medications such as once-weekly injectable semaglutide, insulin degludec, and insulin glargine in various trials of the phase 3 SURPASS clinical trial programme. Compared to placebo, tirzepatide was associated with dose-related mean reductions in $\mathrm{HbA}_{1 \mathrm{c}}$ of $1.00,1.67,1.83$, and $1.89 \%$ with $1,5,10$, and $15 \mathrm{mg}$ doses, respectively [39]. In its cardiovascular outcomes trial, tirzepatide is being compared with dulaglutide $1.5 \mathrm{mg}$, which has a confirmed cardioprotective 
effect. The study is expected to complete by 2024 [40].

\section{GLP1RAs for the Treatment of Obesity}

Obesity is a global challenge for which there are limited therapeutic options. Obesity is a chronic, multifactorial disease that can lead to complications such as cardiovascular diseases (CVDs), T2DM, sleep apnea, and malignancy [41]. Given emerging evidence of the involvement of an abnormal satiety and feeding centre within the brain, there is a need for the development of effective pharmacotherapies or antiobesity medications (AOMs) as an adjunct to lifestyle interventions [42]. While most approved AOMs induce moderate weight loss of up to $10 \%$ [43], the GLP1RA class of drugs and combinations that include them have promising weight reduction benefits (Table 2).

\section{Semaglutide 2.4 mg QW}

The STEP 1 clinical trial has investigated the benefits of once-weekly injections of semaglutide $2.4 \mathrm{mg}$ in the management of people who are obese or overweight [44]. In a double-blind trial of 1961 nondiabetic obese $\left(\geq 30 \mathrm{~kg} / \mathrm{m}^{2}\right)$ or overweight patients $\left(\geq 27 \mathrm{~kg} / \mathrm{m}^{2}\right)$ with coexisting weight-related complications, semaglutide $2.4 \mathrm{mg}$ along with lifestyle modifications led to a $14.9 \%$ reduction in body weight as compared with a $2.4 \%$ reduction with placebo, with an estimated treatment difference of -12.4 percentage points at 68 weeks. The mean reduction in body weight from baseline to week 68 was $-15.3 \mathrm{~kg}$ in the semaglutide group as compared to $-2.6 \mathrm{~kg}$ in the placebo group. $86.4,69.1$, and $50.5 \%$ of the patients on semaglutide $2.4 \mathrm{mg}$ achieved weight losses of $5 \%$ or more, $10 \%$ or more, and $15 \%$ or more, respectively, at the end of 68 weeks. Not only that, patients receiving semaglutide also reported significant improvement in cardiometabolic risk factors and physical functioning as compared to the placebo arm [44]. Transient nausea and diarrhoea were the most common adverse events; these were of mild-to-moderate severity that subsided over time [41-43]. The STEP 2 trial compared semaglutide $2.4 \mathrm{mg}$ versus placebo and semaglutide $1 \mathrm{mg}$ for weight management in a double-blind, double-dummy design in overweight and obese T2DM patients. At the end of 68 weeks, semaglutide $2.4 \mathrm{mg}$ once a week demonstrated a superior and clinically meaningful decrease in body weight compared with placebo, with an estimated treatment difference of 6.2 percentage points $(95 \% \mathrm{CI}-7.3$ to -5.2 ; $p<0.0001)$. A higher number of patients with semaglutide $2.4 \mathrm{mg}$ versus placebo achieved a weight loss of $5 \%$ or more (OR $-4.88,95 \%$ CI 3.58-6.64) [45]. Furthermore, semaglutide $2.4 \mathrm{mg}$ has been investigated versus placebo as an adjunct to intensive behavioural therapy and a low-calorie diet for weight management in 611 overweight and obese patients in the STEP 3 trial. Higher proportions of the patients in the semaglutide $2.4 \mathrm{mg}$ arm achieved weight losses of $5 \%$ or more, $10 \%$ or more, and $15 \%$ or more at the end of 68 weeks: 86.6, 75.3, and 55.8\%, respectively [46].

\section{Liraglutide $3 \mathrm{mg}$}

Liraglutide $3 \mathrm{mg}$ once-daily injection is approved as an AOM. The double-blind 56-week SCALE trial of 3,731 people without T2DM showed that liraglutide $3 \mathrm{mg}$ induced a superior weight reduction of $-8 \%$ as compared to $-2.6 \%$ with placebo in 56 weeks [47]. While the weight loss benefits of liraglutide have been shown to be maintained for 2 years, patients are also able to tolerate the therapy, with only mildto moderate-intensity adverse drug reactions (ADRs) relating to the GIT encountered [48]. A total of five randomized, placebo-controlled trials have been conducted to evaluate the efficacy and safety of liraglutide for weight management. Liraglutide $3 \mathrm{mg}$ as an adjunct to diet and physical activity consistently resulted in a 4-6 kg weight loss, with higher proportions of patients achieving weight losses of at least 5 and $10 \%$ compared with placebo. The comparative data suggest that greater weight loss is achieved with liraglutide compared to orlistat or lorcaserin, but slightly lower weight loss than achieved with phentermine/topiramate [49]. 


\section{Oral Semaglutide $50 \mathrm{mg}$}

Oral semaglutide at a high dose of $50 \mathrm{mg}$ will be examined in a phase 3a trial in the year 2021. With approximately 1000 obese or overweight subjects with comorbidities included in a 68-week-long study, this trial is designed to compare oral semaglutide and placebo in terms of efficacy and safety [50].

\section{Danuglipron}

Apart from its use in the management of T2DM, danuglipron has also been tested in obese and high-BMI patients for its weight loss benefits.

\section{GLP-1 Co-formulations for the Treatment of Obesity}

To achieve better weight-loss efficacy and mitigation of ADRs, combination therapies are now accepted in the management of obesity [51]. GLP1RAs can be combined with other compounds to target multiple signalling pathways involved in energy balance regulation.

GLP1RAs and Amylin Amylin, a peptide cosecreted with insulin from beta cells of the pancreas upon the ingestion of food, is known to regulate appetite, delay gastric emptying, and cause weight loss by acting on amylin receptors in the area postrema [52]. Rodent studies have shown that incremental administration of the amylin/calcitonin receptor agonist salmon calcitonin (sCT) along with liraglutide results in greater reductions in food intake, adipose tissue, and body weight compared to the individual drugs [53].

A novel once-weekly subcutaneous amylin analogue known as AM833 or cagrilintide has been tested in combination with semaglutide QW in diet-induced obese (DIO) rats and mice. In relation to the baseline body weight, DIO rats showed greater weight loss with the combination therapy than with each monotherapy [54]. A 20-week phase 1b RCT testing AM833 with semaglutide $1.2 \mathrm{mg} / 2.4 \mathrm{mg}$ in overweight or obese patients proved that the combination is not only safe and well tolerated but that it also causes substantial weight loss, resulting in a mean percentage body weight reduction of $15-17 \%$ [55]. AM833 at a dose of $4.5 \mathrm{mg}$ achieved weight loss of up to $10.8 \%$ when used as a monotherapy over 26 weeks. Whether it would be able to achieve greater weight loss relative to semaglutide if used as a monotherapy with longer exposure remains to be confirmed [56].

GLP-1/Glucagon Receptor Dual Agonists Glucagon, GLP-1, and oxyntomodulin (OXM) are transcription products of the proglucagon gene. Although all three peptide hormones have a similar source, they have different or opposite physiological roles, which makes them ideal candidates for development as coagonists.

Apart from hyperglycaemia, glucagon receptor stimulation also leads to lipolysis as well as thermogenesis. These two effects would be beneficial for obese patients if glucagon receptor agonism could be performed in the presence of a counteracting drug such as a GLP1RA [57]. A rodent study of GLP-1/glucagon co-agonism demonstrated the restoration of leptin responsiveness in mice maintained on a high-fat diet, leading to $15 \%$ weight loss [58]. The beneficial effects of glucagon on body fat mass, nutrient intake, and energy expenditure are well documented from preclinical and clinical studies $[59,60]$. Further, human phase 1 and 2 trials to investigate the actions of GLP-1/ glucagon receptor dual agonists in obese patients are ongoing. GLP-1 and glucagon have also been used to selectively target G-proteincoupled receptors and to deliver steroid hormones as cargo to specific cell types in preclinical studies. Conjugation of GLP-1 with the glucocorticoid hormone dexamethasone produced striking weight loss through a combination of reduced food intake and increased energy expenditure in obese mice [61]. Peptides with GLP-1/glucagon co-agonist properties are listed in Table 3.

GLP-1/GIP Receptor Dual Agonists Another method of harnessing the catabolic effects of glucagon is to stimulate GIP receptors, which in turn stimulates glucagon secretion. However, this may enhance the diabetogenic potential of glucagon, which is involved in gluconeogenesis and glycogenolysis. Combining GIP with GLP-1 
Table 2 Mean $\mathrm{HbA}_{1 \mathrm{c}}$ reduction and weight loss induced by each GLP1RA and their combinations

\begin{tabular}{lllll}
\hline Drug & $\mathbf{H b A}_{\mathbf{1 c}}$ reduction (\%) & Reference(s) & Weight reduction $(\mathbf{k g})$ & Reference(s) \\
\hline Exenatide BD & $1 / 0.86$ & {$[6,68]$} & $1-2.5$ & {$[6]$} \\
Exenatide ER & 1.28 & {$[68]$} & & \\
Lixisenatide & 0.52 & {$[69]$} & 0.65 & {$[69]$} \\
Albiglutide & 0.66 & {$[70]$} & 0.26 & {$[70]$} \\
Dulaglutide & $1.38 / 1.18$ & {$[68,70]$} & 0.88 & {$[70]$} \\
Liraglutide $1.8 \mathrm{mg}$ & $1.34 / 1.3-1.6$ & {$[14,68,71]$} & 3.24 & {$[71]$} \\
Liraglutide 3 mg & & & $4-6$ & {$[49]$} \\
Semaglutide 1 mg & $1.8 / 1.38$ & {$[17,72,73]$} & $4.5-6.5$ & {$[17,72]$} \\
Semaglutide 2.4 mg & $0.5-1.6$ & {$[45,46]$} & $9.7-16.8$ & {$[45,46,74]$} \\
Oral semaglutide 14 mg & $1.5-1.7$ & {$[75-79]$} & $4.1-4.7$ & {$[75-79]$} \\
Tirzepatide & $1.9-2.2$ & {$[80]$} & $4-5.7$ & {$[80]$} \\
\hline
\end{tabular}

Table 3 Peptides with GLP-1/glucagon co-agonist properties that are under development [81]

\begin{tabular}{lll}
\hline Peptide & Proposed dose frequency & Developer \\
\hline $\begin{array}{l}\text { Preclinical phase } \\
\text { IUB447 }\end{array}$ & Daily s.c. injection & MB-2 LLC \\
MOD-6030/1 & Weekly s.c. injection & Prolor/OPKO Biologics \\
VPD-107 & Weekly s.c. injection & Spitfire Pharma \\
In phase 1 trials & & \\
SAR 425899 & s.c. injection & Sanofi \\
MEDI0382 & s.c. injection & MedImmune \\
HM2525A & Weekly s.c. injection & Hamni Pharmaceuticals \\
ZP2929 & Once-daily s.c. injection & Zealand and Boehringer Ingelheim \\
In phase 2 trials & & Eli Lilly \\
LY2944876/TT-401 & Once-weekly s.c. injection & \\
Not therapeutically useful & & \\
Oxyntomodulin & Short-acting; $t_{1 / 2} \sim 12$ min & \\
\hline
\end{tabular}

would not only reap the weight loss benefits of GLP-1 but also counteract the obesogenic potential of GIP. Potentiation of weight loss has been observed with the combination of GLP-1 and GIP, which shows that there is a distinct set of neurons for the action of GLP-1 and GIP in the ARN [62]. Tirzepatide at doses of 5, 10, and $15 \mathrm{mg}$ is currently being investigated as an AOM in the SURMOUNT clinical trial programme. The SURMOUNT-1 trial is testing the ability of tirzepatide to produce weight loss in people who are overweight or obese. Participants are 
randomized to tirzepatide at doses of 5,10 , or $15 \mathrm{mg}$, and the co-primary endpoints are the percent change in body weight and the proportion of people attaining at least a 5\% reduction in their baseline body weight by week 72. The anticipated study completion date is May 2024 [63]. Tirzepatide has been found to be associated with a marked dose-dependent loss of body weight: a loss of $0.9 \mathrm{~kg}$ was observed with a dose of $1 \mathrm{mg}$, or $11.3 \mathrm{~kg}$ with a dose of $15 \mathrm{mg}$, whereas reductions of $2.7 \mathrm{~kg}$ with dulaglutide and $0.4 \mathrm{~kg}$ with placebo were noted. Over half of the patients receiving the $15 \mathrm{mg}$ dose of tirzepatide developed antibodies to this agent, but these were generally of low titre and did not appear to compromise efficacy [64].

GLP-1/GIP/Glucagon Receptor Triple Agonists As discussed above, glucagon receptor stimulation has a role to play in weight loss by increasing energy expenditure. Combining the effects of GLP-1/GIP and glucagon receptor agonism in a single molecule might have the greatest impact on weight control. The GLP-1 action would cause a reduction in calorie intake and GIP stimulation would potentiate the incretin effect, which would neutralize the diabetogenic action of glucagon [65]. Weight lowering was particularly effective with some triagonists, which may be attributable to the inclusion of glucagon agonism: glucagon exerts a satiety effect and promotes energy expenditure. It is anticipated that the glucose-lowering effects conferred by the GLP-1 and GIP components of the triagonists more than compensate for the effect of glucagon receptor agonism in promoting endogenous glucose production. Further studies are awaited on this concept [66]. HM15211 consists of a novel long-acting glucagon, gastric inhibitory peptide, and glucagonlike peptide 1 (glucagon/GIP/GLP-1) receptor triple full agonist that is chemically conjugated with the constant region of human immunoglobulin via a nonpeptidyl flexible linker. A phase 1, first-in-human (FIH), single ascending dose (SAD) study to confirm the safety and tolerability profiles of HM15211 in healthy obese subjects was completed (NCT03374241) [67].

\section{CONCLUSION}

The present review represents a summary of the latest developments in GLP1RA therapy for the management of T2DM and obesity. Such developments help to improve patient outcomes and patient convenience (through the use of once-weekly dosage forms and oral formulations). In addition to GLP1RA monotherapies, various additional GLP1RA coadministration strategies have demonstrated benefits when used for the treatment of T2DM and obesity. To achieve comprehensive metabolic control, attempts have also been made to target other receptors in concurrence with GLP1RA using a single drug in the form of coagonists or triple agonists. Advances in drug development and the rapidly expanding list of GLP-1-related drugs present a need to create a clinically relevant, pharmacology-based classification of these molecules. The present article fills this void and should remain relevant for the foreseeable future.

\section{ACKNOWLEDGEMENTS}

Compliance with Ethics Guidelines. This article is based on previously conducted studies and does not contain any new studies with human participants or animals performed by any of the authors.

Funding. No funding or sponsorship was received for this study or publication of this article.

Authorship. All named authors meet the International Committee of Medical Journal Editors (ICMJE) criteria for authorship for this article, take responsibility for the integrity of the work as a whole, and have given their approval for this version to be published.

Disclosures. Sanjay Kalra has received speaker fees from Eli Lilly, NovoNordisk and Sanofi and is Editorial Board member of the journal; Saptarshi Bhattacharya has received 
speaker fees from Eli Lilly and NovoNordisk; Nitin Kapoor has nothing to disclose.

Data Availability. Data sharing is not applicable to this manuscript as no datasets were generated or analyzed during the current study.

Open Access. This article is licensed under a Creative Commons Attribution-NonCommercial 4.0 International License, which permits any non-commercial use, sharing, adaptation, distribution and reproduction in any medium or format, as long as you give appropriate credit to the original author(s) and the source, provide a link to the Creative Commons licence, and indicate if changes were made. The images or other third party material in this article are included in the article's Creative Commons licence, unless indicated otherwise in a credit line to the material. If material is not included in the article's Creative Commons licence and your intended use is not permitted by statutory regulation or exceeds the permitted use, you will need to obtain permission directly from the copyright holder. To view a copy of this licence, visit http://creativecommons.org/licenses/by$\mathrm{nc} / 4.0 /$.

\section{REFERENCES}

1. Gupta Y, Kalra S. Choice of glucose-lowering therapy-a metabolic fulcrum-based approach. US Endocrinol. 2015;11(02):79.

2. Aroda VR. A review of GLP-1 receptor agonists: evolution and advancement, through the lens of randomised controlled trials. Diabetes Obes Metab. 2018;20(Suppl 1):22-33.

3. Sheahan KH, Wahlberg EA, Gilbert MP. An overview of GLP-1 agonists and recent cardiovascular outcomes trials. Postgrad Med J. 2020;96(1133): $156-61$.

4. Szayna M, Doyle ME, Betkey JA, Holloway HW, Spencer RGS, Greig NH, Egan JM. Exendin-4 decelerates food intake, weight gain, and fat deposition in Zucker rats. Endocrinology. 2000;141(6): 1936-41.
5. McCormack PL. Exenatide twice daily: a review of its use in the management of patients with type 2 diabetes mellitus. Drugs. 2014;74(3):325-51.

6. Norris SL, Lee N, Thakurta S, Chan BK. Exenatide efficacy and safety: a systematic review. Diabet Med. 2009;26(9):837-46.

7. Jose B, Tahrani AA, Piya MK, Barnett AH. Exenatide once weekly: clinical outcomes and patient satisfaction. Patient Prefer Adherence. 2010;4:313-24.

8. Fineman MS, Mace KF, Diamant M, Darsow T, Cirincione $\mathrm{BB}$, Booker Porter TK, et al. Clinical relevance of anti-exenatide antibodies: safety, efficacy and cross-reactivity with long-term treatment. Diabetes Obes Metab. 2012;14(6):546-54.

9. Ratner RE, Rosenstock J, Boka G. Dose-dependent effects of the once-daily GLP-1 receptor agonist lixisenatide in patients with type 2 diabetes inadequately controlled with metformin: a randomized, double-blind, placebo-controlled trial. Diabet Med. 2010;27(9):1024-32.

10. Lorenz M, Pfeiffer C, Steinstrasser A, Becker RH, Rutten H, Ruus P, et al. Effects of lixisenatide once daily on gastric emptying in type 2 diabetes-relationship to postprandial glycemia. Regul Pept. 2013;185:1-8.

11. Barnett AH. Lixisenatide: evidence for its potential use in the treatment of type 2 diabetes. Core Evid. 2011;6:67-79.

12. Madsen K, Knudsen LB, Agersoe $H$, Nielsen PF, Thøgersen $H$, Wilken M, Johansen NL. Structure-activity and protraction relationship of longacting glucagon-like peptide-1 derivatives: importance of fatty acid length, polarity, and bulkiness. J Med Chem. 2007;50:6126-32.

13. Buse JB, Garber A, Rosenstock J, Schmidt WE, Brett $\mathrm{JH}$, Videbaek $\mathrm{N}$, et al. Liraglutide treatment is associated with a low frequency and magnitude of antibody formation with no apparent impact on glycemic response or increased frequency of adverse events: results from the Liraglutide Effect and Action in Diabetes (LEAD) trials. J Clin Endocrinol Metab. 2011;96(6):1695-702.

14. Rigato M, Fadini GP. Comparative effectiveness of liraglutide in the treatment of type 2 diabetes. Diabetes Metab Syndr Obes. 2014;7:107-20.

15. Marso SP, Daniels GH, Brown-Frandsen K, Kristensen P, Mann JF, Nauck MA, et al. Liraglutide and cardiovascular outcomes in type 2 diabetes. N Engl J Med. 2016;375(4):311-22. 
16. Knudsen LB, Lau J. The discovery and development of liraglutide and semaglutide. Front Endocrinol (Lausanne). 2019;10:155.

17. Goldenberg RM, Steen O. Semaglutide: review and place in therapy for adults with type 2 diabetes. Can J Diabetes. 2019;43(2):136-45.

18. Marso SP, Bain SC, Consoli A, Eliaschewitz FG, Jodar E, Leiter LA, et al. Semaglutide and cardiovascular outcomes in patients with type 2 diabetes. N Engl J Med. 2016;375(19):1834-44.

19. Vilsboll T, Bain SC, Leiter LA, Lingvay I, Matthews $\mathrm{D}$, Simo R, et al. Semaglutide, reduction in glycated haemoglobin and the risk of diabetic retinopathy. Diabetes Obes Metab. 2018;20(4):889-97.

20. Glaesner W, Vick AM, Millican R, Ellis B, Tschang $\mathrm{SH}$, Tian $\mathrm{Y}$, et al. Engineering and characterization of the long-acting glucagon-like peptide-1 analogue LY2189265, an Fc fusion protein. Diabetes Metab Res Rev. 2010;26(4):287-96.

21. Zhang L, Zhang M, Zhang Y, Tong N. Efficacy and safety of dulaglutide in patients with type 2 diabetes: a meta-analysis and systematic review. Sci Rep. 2016;6:18904.

22. Kugler AJ, Thiman ML. Efficacy and safety profile of once-weekly dulaglutide in type 2 diabetes: a report on the emerging new data. Diabetes Metab Syndr Obes. 2018;11:187-97.

23. Gerstein HC, Colhoun HM, Dagenais GR, Diaz R, Lakshmanan M, Pais P, Probstfield J, Riesmeyer JS, Riddle MC, Rydén L, Xavier D, Atisso CM, Dyal L, Hall S, Rao-Melaci P. Dulaglutide and cardiovascular outcomes in type 2 diabetes (REWIND): a double-blind, randomised placebo-controlled trial. Lancet. 2019;394:121-30.

24. Rendell MS. Albiglutide: a unique GLP-1 receptor agonist. Expert Opin Biol Ther. 2016;16(12): 1557-69.

25. Lund A, Knop FK, Vilsboll T. Glucagon-like peptide1 receptor agonists for the treatment of type 2 diabetes: differences and similarities. Eur J Intern Med. 2014;25(5):407-14.

26. Avgerinos I, Michailidis T, Liakos A, Karagiannis T, Matthews DR, Tsapas A, et al. Oral semaglutide for type 2 diabetes: a systematic review and metaanalysis. Diabetes Obes Metab. 2020;22(3):335-45.

27. Clinicaltrial.gov. Novo Nordisk A/S. Identifier NCT04707469. Research study to compare three doses of semaglutide tablets taken once daily in people with type 2 diabetes (PIONEER PLUS). 13 Jan 2021. Available from: https://clinicaltrials.gov/ct2/ show/NCT04707469. Accessed 2 May 2021.
28. Griffith DA, Edmonds DJ, Fortin J-P, Kalgutkar AS, Kuzmiski JB, Loria PM, et al. A small-molecule oral agonist of the human glucagon-like peptide-1 receptor. bioRxiv. 2020:319483.

29. Businesswire. Pfizer Investor Day features significant number of pipeline advances for COVID-19 programs and across numerous therapeutic areas. 2020. Available from: https://www.businesswire. com/news/home/20200915006138/en/. Accessed 15 Sept 2020.

30. Kalra S, Saboo B. Exenatide implant therapy in diabetes. Recent Adv Endocrinol. 2018;68(10): 1538-40.

31. Schwartz SS, Epstein S, Corkey BE, Grant SF, Gavin JR 3rd, Aguilar RB. The time is right for a new classification system for diabetes: rationale and implications of the beta-cell-centric classification schema. Diabetes Care. 2016;39(2):179-86.

32. Abdul-Ghani MA, Puckett C, Triplitt C, Maggs D, Adams J, Cersosimo E, et al. Initial combination therapy with metformin, pioglitazone and exenatide is more effective than sequential add-on therapy in subjects with new-onset diabetes. Results from the Efficacy and Durability of Initial Combination Therapy for Type 2 Diabetes (EDICT): a randomized trial. Diabetes Obes Metab. 2015;17(3): 268-75.

33. Anderson SL, Trujillo JM. Basal insulin use with GLP-1 receptor agonists. Diabetes Spectr. 2016;29(3):152-60.

34. Blonde L, Anderson JE, Chava P, Dendy JA. Rationale for a titratable fixed-ratio co-formulation of a basal insulin analog and a glucagon-like peptide 1 receptor agonist in patients with type 2 diabetes. Curr Med Res Opin. 2019;35(5):793-804.

35. Rosenstock J, Diamant M, Aroda V, Silvestre L, Souhami E, Zhou T, et al. Efficacy and safety of LixiLan, a titratable fixed-ratio combination of lixisenatide and insulin glargine, versus insulin glargine in type 2 diabetes inadequately controlled on metformin monotherapy: the LixiLan proof-ofconcept randomized trial. Diabetes Care. 2016;39: 1579-86.

36. Price H, Bluher M, Prager R, Phan TM, Thorsted BL, Schultes B, et al. Use and effectiveness of a fixedratio combination of insulin degludec/liraglutide (IDegLira) in a real-world population with type 2 diabetes: results from a European, multicentre, retrospective chart review study. Diabetes Obes Metab. 2018;20(4):954-62.

37. Greig SL, Scott LJ. Insulin degludec/liraglutide: a review in type 2 diabetes. Drugs. 2015;75(13): 1523-34. 
38. DoctoRx. Seeking Alpha: Novo Nordisk's insulin products, pipeline may energize the stock. 2020. Available from: https://seekingalpha.com/article/ 4323697-novo-nordisks-insulin-products-pipelinemay-energize-stock. Accessed 2 May 2021.

39. Clinicaltrial.gov. Eli Lilly and Company. Identifier: NCT03954834. A study of tirzepatide (LY3298176) in participants with type 2 diabetes not controlled with diet and exercise alone (SURPASS-1). May 17, 2019. Available from: https://clinicaltrials.gov/ct2/ show/NCT03954834. Accessed 2 May 2021.

40. Clinicaltrial.gov. Eli Lilly and Company. Identifier: NCT04255433. A study of tirzepatide (LY3298176) compared with dulaglutide on major cardiovascular events in participants with type 2 diabetes (SURPASS-CVOT). 5 Feb 2020. Available from: https:// clinicaltrials.gov/ct2/show/NCT04255433. Accessed 2 May 2021.

41. Fruhbeck G, Busetto L, Dicker D, Yumuk V, Goossens GH, Hebebrand J, et al. The ABCD of obesity: an EASO position statement on a diagnostic term with clinical and scientific implications. Obes Facts. 2019;12(2):131-6.

42. Acosta A, Abu Dayyeh BK, Port JD, Camilleri M. Recent advances in clinical practice challenges and opportunities in the management of obesity. Gut. 2014;63(4):687-95.

43. Bray GA, Frühbeck G, Ryan DH, Wilding JPH. Management of obesity. Lancet. 2016;387(10031): 1947-56.

44. Kushner RF, Calanna S, Davies M, Dicker D, Garvey WT, Goldman B, et al. Semaglutide $2.4 \mathrm{mg}$ for the treatment of obesity: key elements of the STEP trials 1 to 5. Obesity (Silver Spring). 2020;28(6):1050-61.

45. Davies M, Færch L, Jeppesen OK, Pakseresht A, Pedersen SD, Perreault L, et al. Semaglutide $2.4 \mathrm{mg}$ once a week in adults with overweight or obesity, and type 2 diabetes (STEP 2): a randomised, doubleblind, double-dummy, placebo-controlled, phase 3 trial. Lancet. 2021;397(10278):971-84.

46. Wadden TA, Bailey TS, Billings LK, Davies M, Frias JP, Koroleva A, et al. Effect of subcutaneous semaglutide vs placebo as an adjunct to intensive behavioral therapy on body weight in adults with overweight or obesity: the STEP 3 randomized clinical trial. JAMA. 2021;325(14):1403-13.

47. Pi-Sunyer X, Astrup A, Fujioka K, Greenway F, Halpern A, Krempf $M$, et al. A randomized, controlled trial of $3.0 \mathrm{mg}$ of liraglutide in weight management. N Engl J Med. 2015;373(1):11-22.
48. Scott LJ. Liraglutide: a review of its use in the management of obesity. Drugs. 2015;75(8): 899-910.

49. Mehta A, Marso SP, Neeland IJ. Liraglutide for weight management: a critical review of the evidence. Obes Sci Pract. 2017;3(1):3-14.

50. Novo Nordisk. Novo Nordisk to initiate phase 3a development in obesity with oral semaglutide. Company announcement no. 25/2021. Bagsværd: Novo Nordisk; 2021.

51. Wilding JP. Combination therapy for obesity. J Psychopharmacol. 2017;31(11):1503-8.

52. Aronne L, Fujioka K, Aroda V, Chen K, Halseth A, Kesty NC, et al. Progressive reduction in body weight after treatment with the amylin analog pramlintide in obese subjects: a phase 2 , randomized, placebo-controlled, dose-escalation study. J Clin Endocrinol Metab. 2007;92(8):2977-83.

53. Liberini CG, Koch-Laskowski K, Shaulson E, McGrath LE, Lipsky RK, Lhamo R, et al. Combined amylin/GLP-1 pharmacotherapy to promote and sustain long-lasting weight loss. Sci Rep. 2019;9(1): 8447.

54. John LM, Kruse T, Raun K. Preclinical weight loss efficacy of AM833 in combination with semaglutide in rodent models of obesity. In: ENDO 2021-103rd Annual Meeting of the Endocrine Society, 2021 Mar 20-23.

55. Enebo LB. Safety and tolerability of concomitant administration of multiple dose AM833 with semaglutide $2.4 \mathrm{mg}$ for weight management. ENDO 2021-103rd Annual Meeting of the Endocrine Society, 2021 Mar 20-23.

56. Novo Nordisk Inc. Novo Nordisk Inc. successfully completes AM833 phase 2 trial and phase 1 combination trial with AM833 and semaglutide in obesity. Press release from 18 Jun 2020. Available from: https://www.novonordisk.com/content/ nncorp/global/en/news-and-media/news-and-irmaterials/news-details.html?id $1 / 4274$. $>$. Accessed 2 May 2021.

57. Sanchez-Garrido MA, Brandt SJ, Clemmensen C, Muller TD, DiMarchi RD, Tschop MH. GLP-1/glucagon receptor co-agonism for treatment of obesity. Diabetologia. 2017;60(10):1851-61.

58. Clemmensen C. GLP-1/glucagon coagonism restores leptin responsiveness in obese mice chronically maintained on an obesogenic diet. Diabetes. 2014;63:1422-7.

59. Habegger KM, Heppner KM, Geary N, Bartness TJ, DiMarchi R, Tschop MH. The metabolic actions of 
glucagon revisited. Nat Rev Endocrinol. 2010;6(12): 689-97.

60. Habegger KM, Stemmer K, Cheng C, Müller TD, Heppner KM, Ottaway N, Holland J, Hembree JL, Smiley D, Gelfanov V, Krishna R. Fibroblast growth factor 21 mediates specific glucagon actions. Diabetes. 2013;62:1453-63.

61. Quarta C, Clemmensen C, Zhu Z, Yang B, Joseph SS, Lutter D, et al. Molecular integration of incretin and glucocorticoid action reverses immunometabolic dysfunction and obesity. Cell Metab. 2017;26(4):620-632.e6.

62. NamKoong C, Kim MS, Jang BT, Lee YH, Cho YM, Choi HJ. Central administration of GLP-1 and GIP decreases feeding in mice. Biochem Biophys Res Commun. 2017;490(2):247-52.

63. Clinicaltrials.gov. Eli Lilly and Company. Identifier: NCT04184622. A study of tirzepatide (LY3298176) in participants with obesity or overweight (SURMOUNT-1). 19 Dec 2019. Available from: https:// clinicaltrials.gov/ct2/show/NCT04184622. Accessed 2 May 2021.

64. Frias JP, Nauck MA, Van J, Kutner ME, Cui X, Benson C, et al. Efficacy and safety of LY3298176, a novel dual GIP and GLP-1 receptor agonist, in patients with type 2 diabetes: a randomised, placebo-controlled and active comparator-controlled phase 2 trial. Lancet. 2018;392(10160):2180-93.

65. Finan B, Yang B, Ottaway N, Smiley DL, Ma T, Clemmensen $\mathrm{C}$, et al. A rationally designed monomeric peptide triagonist corrects obesity and diabetes in rodents. Nat Med. 2015;21(1):27-36.

66. Gault VA, Bhat VK, Irwin N, Flatt PR. A novel glucagon-like peptide-1 (GLP-1)/glucagon hybrid peptide with triple-acting agonist activity at glucosedependent insulinotropic polypeptide, GLP-1, and glucagon receptors and therapeutic potential in high fat-fed mice. J Biol Chem. 2013;288(49): 35581-91.

67. Clinicaltrials.gov. Hanmi Pharmaceutical Company Limited. Identifier: NCT03374241. A first-in-human study to evaluate the safety, tolerability, pharmacokinetics and pharmacodynamics of HM15211. 15 Dec 2017. Available from: https://clinicaltrials.gov/ ct2/show/NCT03374241. Accessed 2 May 2021.

68. Fahrbach JL, Fu H, Shurzinske L, Skrivanek Z, Martin S. Network meta-analysis accurately predicted the outcome of a subsequent randomised trial comparing once weekly dulaglutide $1.5 \mathrm{mg}$ and once daily liraglutide $1.8 \mathrm{mg}$. Int J Clin Pract. 2016;70(3):218-21.
69. Schmidt LJ. A systematic review and meta-analysis of the efficacy of lixisenatide in the treatment of patients with type 2 diabetes. Diabetes Obes Metab. 2014;16:769-79.

70. Karagiannis T. Efficacy and safety of once-weekly glucagon-like peptide 1 receptor agonists for the management of type 2 diabetes: a systematic review and meta-analysis of randomized controlled trials. Diabetes Obes Metab. 2015;17:1065-74.

71. Montanya E, Sesti G. A review of efficacy and safety data regarding the use of liraglutide, a once-daily human glucagon-like peptide 1 analogue, in the treatment of type 2 diabetes mellitus. Clin Ther. 2009;31(11):2472-88.

72. Kalra S, Sahay R. A review on semaglutide: an oral glucagon-like peptide 1 receptor agonist in management of type 2 diabetes mellitus. Diabetes Ther. 2020;11(9):1965-82.

73. Andreadis $\mathrm{P}$, Karagiannis T, Malandris K, Avgerinos I, Liakos A, Manolopoulos A, et al. Semaglutide for type 2 diabetes mellitus: a systematic review and meta-analysis. Diabetes Obes Metab. 2018;20(9): 2255-63.

74. Wilding JPH, Batterham RL, Calanna S, Davies M, Van Gaal LF, Lingvay I, et al. Once-weekly semaglutide in adults with overweight or obesity. N Engl J Med. 2021;384(11):989.

75. Yamada Y, Katagiri H, Hamamoto Y, Deenadayalan $S$, Navarria A, Nishijima $K$, et al. Dose-response, efficacy, and safety of oral semaglutide monotherapy in Japanese patients with type 2 diabetes (PIONEER 9): a 52-week, phase 2/3a, randomised, controlled trial. Lancet Diabetes Endocrinol. 2020;8(5):377-91.

76. Rosenstock J, Allison D, Birkenfeld AL, Blicher TM, Deenadayalan S, Jacobsen JB, et al. Effect of additional oral semaglutide vs sitagliptin on glycated hemoglobin in adults with type 2 diabetes uncontrolled with metformin alone or with sulfonylurea: the PIONEER 3 randomized clinical trial. JAMA. 2019;321(15):1466-80.

77. Rodbard HW, Rosenstock J, Canani LH, Deerochanawong C, Gumprecht J, Lindberg SO, et al. Oral semaglutide versus empagliflozin in patients with type 2 diabetes uncontrolled on metformin: the PIONEER 2 trial. Diabetes Care. 2019;42(12): 2272-81.

78. Pratley R, Amod A, Hoff ST, Kadowaki T, Lingvay I, Nauck M, et al. Oral semaglutide versus subcutaneous liraglutide and placebo in type 2 diabetes (PIONEER 4): a randomised, double-blind, phase 3a trial. The Lancet. 2019;394(10192):39-50. 
79. Aroda VR, Rosenstock J, Terauchi Y, Altuntas Y, Lalic NM, Morales Villegas EC, et al. PIONEER 1: randomized clinical trial of the efficacy and safety of oral semaglutide monotherapy in comparison with placebo in patients with type 2 diabetes. Diabetes Care. 2019;42(9):1724-32.

80. Frias JP, Nauck MA, Van J, Benson C, Bray R, Cui X, et al. Efficacy and tolerability of tirzepatide, a dual glucose-dependent insulinotropic peptide and glucagon-like peptide- 1 receptor agonist in patients with type 2 diabetes: a 12-week, randomized, double-blind, placebo-controlled study to evaluate different dose-escalation regimens. Diabetes Obes Metab. 2020;22(6):938-46.

81. Soni H. Peptide-based GLP-1/glucagon co-agonists: a double-edged sword to combat diabesity. Med Hypotheses. 2016;95:5-9. 